\title{
Geometric control of the magnetization reversal in antidot lattices with perpendicular magnetic anisotropy
}

\author{
Joachim Gräfe, ${ }^{*}$ Markus Weigand, Nick Träger, Gisela Schütz, and Eberhard J. Goering ${ }^{\dagger}$ \\ Max Planck Institute for Intelligent Systems, Stuttgart, Germany \\ Maxim Skripnik and Ulrich Nowak \\ Department of Physics, Universität Konstanz, Konstanz, Germany \\ Felix Haering and Paul Ziemann \\ Institute of Solid State Physics, Ulm University, Ulm, Germany \\ Ulf Wiedwald \\ Faculty of Physics and Center for Nanointegration (CENIDE), University of Duisburg-Essen, Duisburg, Germany \\ (Received 21 January 2016; revised manuscript received 23 February 2016; published 28 March 2016)
}

\begin{abstract}
While the magnetic properties of nanoscaled antidot lattices in in-plane magnetized materials have widely been investigated, much less is known about the microscopic effect of hexagonal antidot lattice patterning on materials with perpendicular magnetic anisotropy. By using a combination of first-order reversal curve measurements, magnetic x-ray microscopy, and micromagnetic simulations we elucidate the microscopic origins of the switching field distributions that arise from the introduction of antidot lattices into out-of-plane magnetized GdFe thin films. Depending on the geometric parameters of the antidot lattice we find two regimes with different magnetization reversal processes. For small antidots, the reversal process is dominated by the exchange interaction and domain wall pinning at the antidots drives up the coercivity of the system. On the other hand, for large antidots the dipolar interaction is dominating which leads to fragmentation of the system into very small domains that can be envisaged as a basis for a bit patterned media.
\end{abstract}

DOI: 10.1103/PhysRevB.93.104421

\section{INTRODUCTION}

Periodic arrangements of holes, so called antidot lattices, in thin magnetic films have been broadly investigated in various host materials. In the dynamic regime these nanostructures act as magnonic crystals, and they can be envisaged as spin wave filters and spin wave guides [1-3]. On the other hand, antidot lattices are proposed as a type of bit patterned media that can overcome the superparamagnetic limit as there are no isolated islands [4-6]. Furthermore, antidot lattices exhibit a multitude of novel phenomena like artificial spin ice [7-9] and spin glass [10], and pairs of magnetic monopoles [11].

Mainly, antidot lattices are investigated in magnetic thin films with in-plane anisotropy where the strong influence of the nanostructures due to emergent demagnetization fields at the hole edges is obvious [12]. This is widely used to tune the magnetic anisotropy [13-15] and coercivity [16-18] of these thin films, and the microscopic origin is well established in literature [15,19-24]. To gain an understanding of the processes involved in the magnetization reversal a multitude of approaches have been combined, namely, micromagnetic simulations [15,19,20], magneto-optical Kerr effect (MOKE)

\footnotetext{
*graefe@is.mpg.de

${ }^{\dagger}$ goering@is.mpg.de
}

Published by the American Physical Society under the terms of the Creative Commons Attribution 3.0 License. Further distribution of this work must maintain attribution to the author(s) and the published article's title, journal citation, and DOI. measurements [23,24], photo electron emission microscopy (PEEM) with X-ray circular dichroism (XMCD) contrast [21], scanning X-ray microscopy (SXM) with XMCD contrast [24], and first-order reversal curve (FORC) measurements $[19,22,24]$.

Furthermore, it has been shown that nanoscaled antidot lattices can significantly reduce or overcome the thin film shape anisotropy and result in a perpendicular easy axis $[4,21]$ or perpendicular deflection of the magnetization [12]. However, only a few studies used materials with a perpendicular magnetic anisotropy as host films for the antidot lattices $[25,26]$ and the microscopic interaction of the nanostructures has not been elucidated so far.

FORC measurements are a valuable tool to quantitatively investigate these interactions without the need for high lateral resolution [27]. Therefore, the FORC method is increasingly used for investigations of geomagnetism [28-30], thin film magnetism [31], and magnetic nanostructures [19,24]. The FORC density represents irreversible magnetization reversal processes that are separated by their coercive $H_{c}$ and interaction $H_{u}$ field values. The separation of the individual switching events is achieved by measuring multiple minor hysteresis loops instead of using spatial resolution to distinguish them [27]. Unfortunately, the reliable interpretation of the FORC distribution as a distribution of Preisach hysterons is not always possible [19,32,33]. Therefore, the initial combination of FORC measurements with a highly resolving method like x-ray microscopy in real space, or x-ray and neutron scattering in reciprocal space are frequently used to support the microscopic interpretation of FORC distributions [24,34,35]. 
Here, we describe the influence of nanoscaled antidot structuring with different geometries on the magnetic properties of soft-magnetic GdFe thin films with perpendicular magnetic anisotropy. Furthermore, we shine light on the microscopic processes that are responsible for the change in magnetic properties by a combination of FORC measurements, SXM imaging, and micromagnetic simulations.

\section{METHODS}

The antidot lattices were produced by nanosphere lithography using commercial polystyrene (PS) nanospheres (Invitrogen) with a mean diameter of $210 \pm 20 \mathrm{~nm}$ as starting material [36,37]. Close-packed monolayers of these PS spheres are deposited on $\mathrm{Si}_{3} \mathrm{~N}_{4}(500 \mathrm{~nm}$, membranes $) / \mathrm{Si}(100)$ substrates by dip coating at an extraction velocity of $10 \mu \mathrm{s}^{-1}$ and an angle of $60^{\circ}$ between the substrate surface and the air-water interface. Details of this preparation procedure can be found elsewhere [8,15]. The deposited PS spheres were etched by an oxygen plasma (DC bias: $-85 \mathrm{~V}$ ) to reduce their diameter to $80 \pm 20 \mathrm{~nm}$ or, alternatively, to $160 \pm 20 \mathrm{~nm}$. It has been shown previously that the etching process does not affect the spherical shape of the PS particles [36,37]. On top of these templates $\mathrm{GdFe}[0.36 / 0.36 \mathrm{~nm}]$ multilayer films were deposited with a $2 \mathrm{~nm} \mathrm{Al}$ capping layer under UHV conditions by ion beam sputtering [38]. Finally, the PS spheres including their metal caps were removed by chemomechanical polishing. The total thin film thickness was adjusted from $29 \mathrm{~nm}(40 \times \mathrm{GdFe})$ to $45 \mathrm{~nm}(60 \times \mathrm{GdFe})$ depending on the PS sphere size after etching to allow for their complete removal $[12,24]$. Fe and Gd are generally subjected to oxidation. Although the initial oxidation rate of pure $\mathrm{Fe}$ is very high, oxide formation quickly saturates forming a passivated surface [39]. Degradation of the GdFe multilayer system would lead to a loss of perpendicular magnetic anisotropy, which was not observed during patterning or storage. As the perpendicular anisotropy of the antidot lattice samples was unaffected over the course of several weeks, we suppose that a very thin oxide layer formed on the hole rims and prevented further oxidation.

MOKE based FORC measurements were conducted with a Durham Magneto Optics NanoMOKE3 equipped with an air-cooled vector electromagnet, capable of generating inplane fields up to $120 \mathrm{mT}$. Measurements were performed in polar geometry and the laser beam was directed at the sample with an incidence angle of $90^{\circ}$. For each FORC measurement 600 individual minor loops with different reversal fields $H_{r}$ were acquired, each averaged 10 times at a cycling rate of $0.35 \mathrm{~Hz}$, thus, resulting in a total of 360.000 data points. Here, the FORC density is both influenced by the amount of magnetization change and the degree of irreversibility of the process leading to this magnetization change, i.e., the probability of a stochastic magnetization reversal process being irreversible affects the FORC density [24]. FORC density calculations from the acquired minor loops were done using customized MATLAB code. This was numerically achieved by fitting $[30,40]$ a part of the $M\left(H, H_{r}\right)$ data to

$$
M\left(H, H_{r}\right)=a_{1}+a_{2} H+a_{3} H^{2}+a_{4} H_{r}+a_{5} H_{r}^{2}-2 a_{6} H H_{r}
$$

and extracting the parameter $a_{6}$ as FORC density $\rho$ :

$$
\rho\left(H, H_{r}\right)=-\frac{1}{2} \frac{\partial^{2} M\left(H, H_{r}\right)}{\partial H \partial H_{r}}=a_{6} .
$$

Subsequently, the measurement axes $H$ and $H_{r}$ were transformed into $H_{c}$ and $H_{u}$ axes according to

$$
H_{u}=\frac{1}{2}\left(H+H_{r}\right)
$$

and

$$
H_{c}=\frac{1}{2}\left(H-H_{r}\right) \text {. }
$$

After smoothing this processing scheme lead to FORC distributions with a minimum resolution of $\geqslant 0.5 \mathrm{mT}$ and a baseline resolution of $\leqslant 3 \mathrm{mT}$. Before optimizing the applied smoothing factor, the FORC distribution was inspect at low smoothing factors to exclude loss of information by smoothing. Further details about the acquisition scheme and the data evaluation can be found elsewhere $[12,40]$. In the following $H$ fields are expressed in units of $\mu_{0} \cdot H$ implicitly for convenience.

SXM measurements were conducted at the MPI IS operated MAXYMUS end station at the UE46-PGM2 beam line at the BESSY II synchrotron radiation facility. The samples were illuminated under normal incidence by circularly polarized light in an applied out-of-plane field of up to $240 \mathrm{mT}$ that was generated by a set of four rotatable permanent magnets [41]. The photon energy was set to the absorption maximum of the Fe $L_{3}$ edge to get optimal XMCD contrast for imaging. The transmission through the holes, where there is no material and thus no X-ray absorption, other than from the $\mathrm{Si}_{3} \mathrm{~N}_{4}$ membrane, was used as an internal $I_{0}$ intensity reference to normalize the measured intensities to the incident beam intensity. Intensities were locally averaged using a Gaussian filter in ImageJ [42]. Images taken at different photon helicities were registered using ImageJ with TurboReg [43] to align the individual images [12,24]

Micromagnetic simulations were conducted using a custom implementation of the Landau-Lifshitz-Bloch equation based on OpenCL using graphics processing units to solve the equation of motion [44]. Finite temperatures were implemented as normally distributed random thermal noise [45]. The custom code was verified against $\mu \mathrm{MAG}$ standard problems 3 and 4 [46]. The antidot lattice was discretized into $14 \times 14 \times 14 \mathrm{~nm}^{3}$ cells and as material parameters the saturation magnetization at zero temperature $M_{0}=3.72 \times 10^{5} \mathrm{~A} \mathrm{~m}^{-1}$, the uniaxial anisotropy constant $K_{u}=1.2 \times 10^{5} \mathrm{~J} \mathrm{~m}^{-3}$, and the exchange stiffness at $300 \mathrm{~K} A(300 \mathrm{~K})=7.78 \times 10^{-11} \mathrm{~J} \mathrm{~m}^{-1}$ were assumed [46]. Additionally, 5\% of the macrospins were given an arbitrary magnetization and orientation to include some irregularity of the real samples into the simulations.

\section{RESULTS AND DISCUSSION}

The general layout of antidot lattices with hexagonal symmetry is shown in Fig. 1, indicating that the nanosphere lithography can yield large uniform structural domains with only a few local lattice defects.

The major hysteresis loops for two different antidot lattice geometries and an unstructured thin film are shown in Fig. 2. Both antidot lattices feature the same antidot spacing 


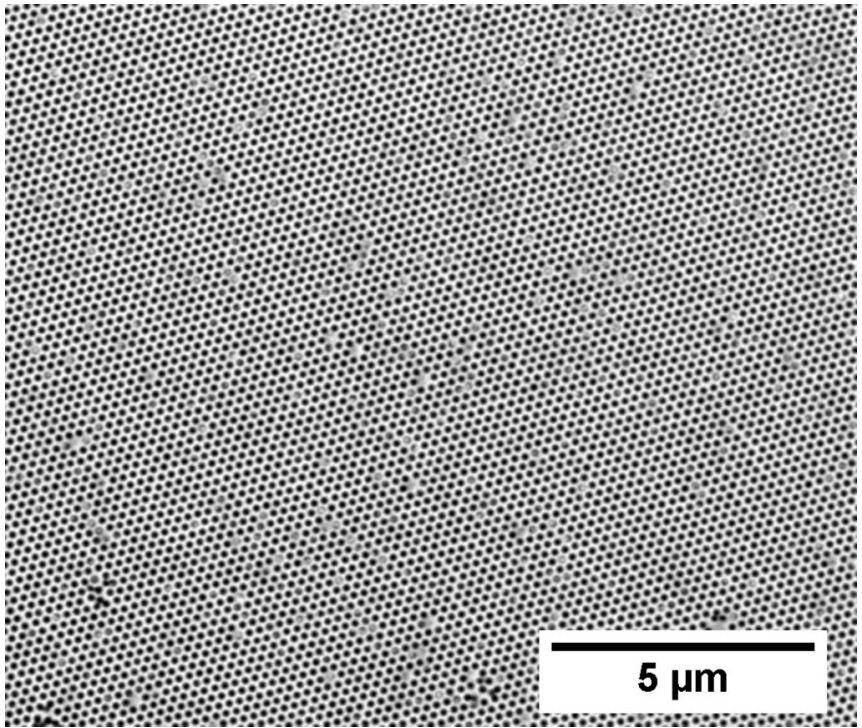

FIG. 1. SEM image of a nanoscaled hexagonal antidot lattice (antidot spacing $a=210 \mathrm{~nm}$ and diameter $d=160 \mathrm{~nm}$ ) in a thin GdFe film.

$a=210 \mathrm{~nm}$, but have different antidot diameters $d$ of $80 \mathrm{~nm}$ and $160 \mathrm{~nm}$, respectively. To compensate for the different PS sphere lithography masks, the antidot lattices were prepared in films with different numbers of multilayers $(40 \times \mathrm{GdFe}$ and $60 \times \mathrm{GdFe}$ respectively), however, there was no appreciable difference in the coercivity of the two host films. While the unstructured thin film exhibits a sharp magnetization reversal with high remanence and a steep jump in the hysteresis loop at the coercive field, resulting in full magnetization reversal, both antidot lattices show similar major hysteresis loops with a reduced remanence and a gradual change of magnetization during reversal. This already suggests that there is a distribution of microscopic processes with different

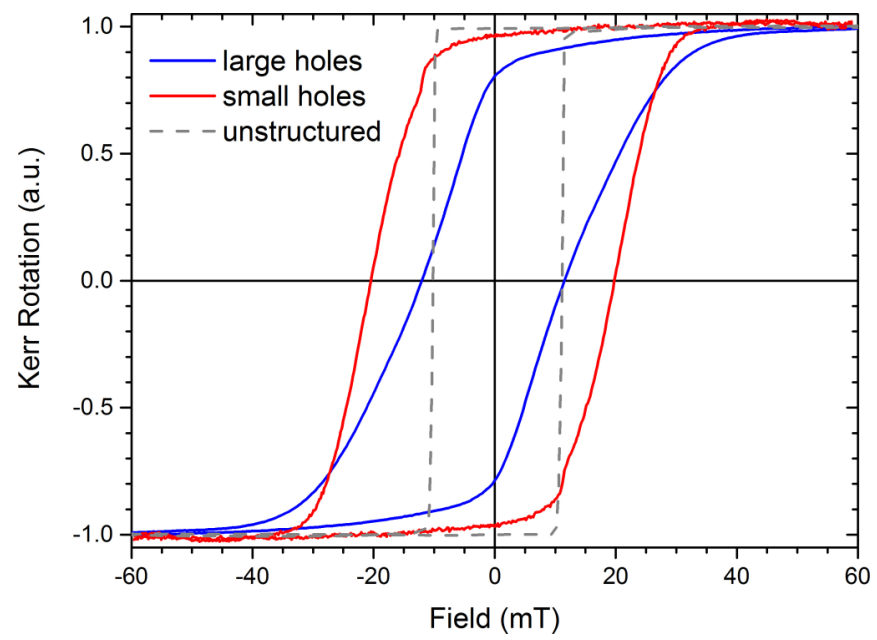

FIG. 2. Major hysteresis loops of GdFe thin films hosting antidot lattices with large (antidot spacing $a=210 \mathrm{~nm}$ and diameter $d=160 \mathrm{~nm}$ ) and small (antidot spacing $a=210 \mathrm{~nm}$ and diameter $d=80 \mathrm{~nm}$ ) holes. The hysteresis loop of an unstructured GdFe thin film is shown for comparison as a dashed line. switching fields. While the saturation field of both antidot lattices is strongly increased, it is noteworthy that the coercive field of the unstructured material is still reflected in the hysteresis loops. For the large holes the overall coercive field remains unchanged, and for the small holes there is a small jump in the hysteresis loop at the original coercive field while the overall coercivity is increased. The latter is in agreement with previous findings of Tripathy and Adeyeye [25] who observe magnetization reversal at the coercive field of unstructured film and a switching field distribution at higher fields. However, the origin of the switching field distribution and the microscopic interactions within the antidot lattices have not been described in literature. In the following, we discuss these properties of the two antidot lattice geometries using a combination of FORC measurements, SXM imaging and micromagnetic simulations to gain a complete microscopic understanding.

\section{A. Antidot lattices with large holes}

For the hexagonal antidot lattice with a large hole diameter (antidot spacing $a=210 \mathrm{~nm}$ and diameter $d=160 \mathrm{~nm}$ ) the FORC diagram is shown in Fig. 3 with the major hysteresis loop shown as inset for comparison. It is noteworthy that the magnetic thin film is narrowed down to a width of $50 \mathrm{~nm}$ between two adjacent holes in this geometry. In general such a major loop results from a distribution of switching fields for all microscopic processes constituting the magnetization reversal. This switching field distribution can either be dominated by a broad distribution in interaction fields or a broad distribution in coercive fields. From the major hysteresis loop alone these origins of the switching field distribution cannot be distinguished. However, from the FORC diagram it is obvious that for the geometry with large holes the former is dominating. The distribution of coercive fields is rather narrow and centered at $10 \mathrm{mT}$, thus, the coercive field is unchanged from the unstructured material for all irreversible switching events.

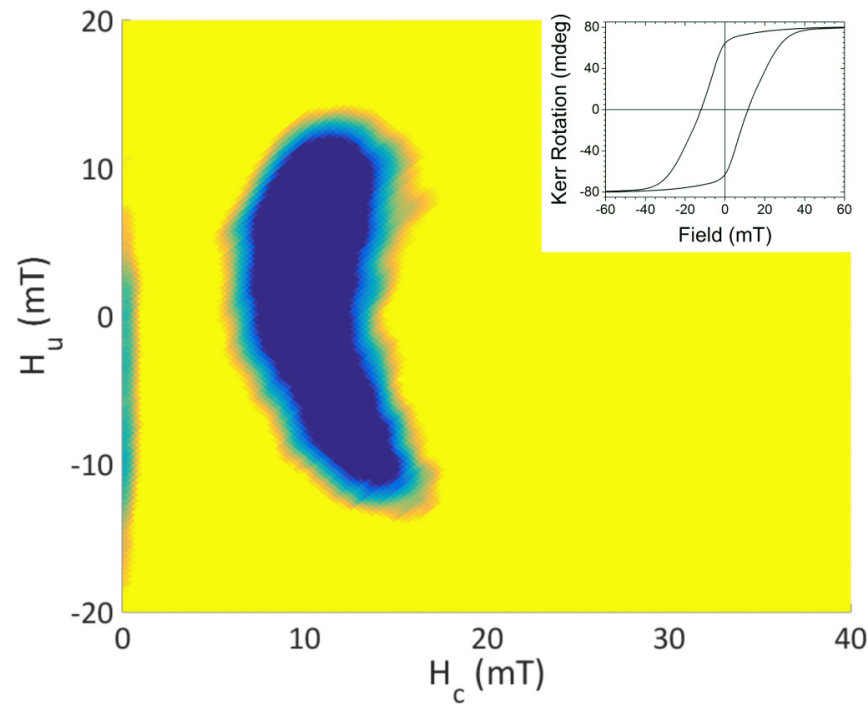

FIG. 3. FORC density of a hexagonal antidot lattice (antidot spacing $a=210 \mathrm{~nm}$ and diameter $d=160 \mathrm{~nm}$ ) in a $45 \mathrm{~nm}$ thin GdFe film. The major hysteresis loop is shown as inset. 


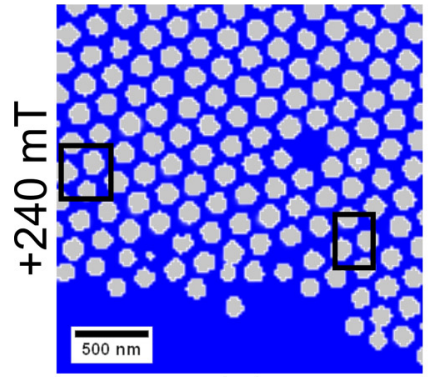

(a)

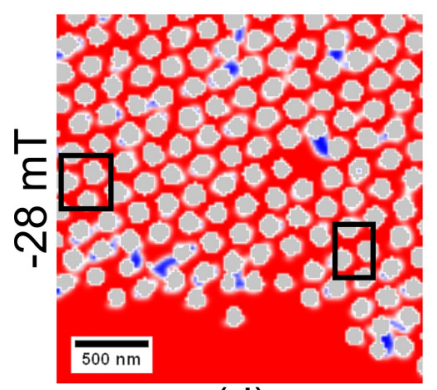

(d)

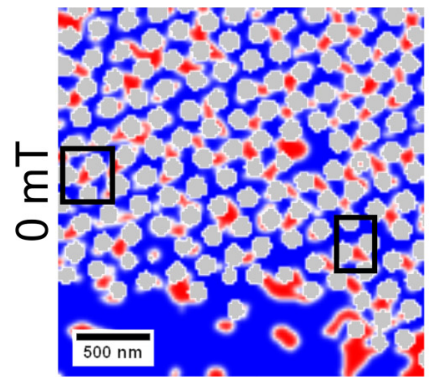

(b)

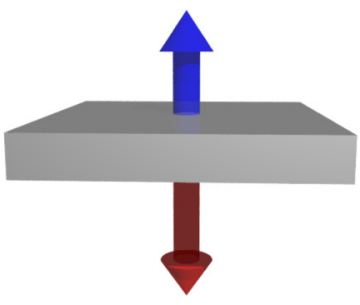

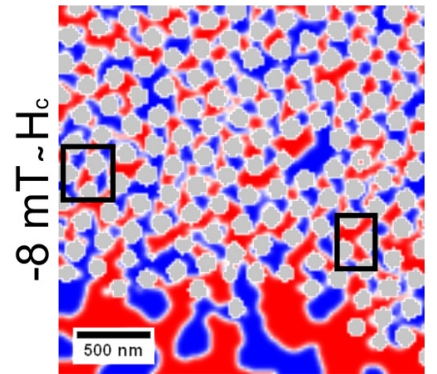

(c)

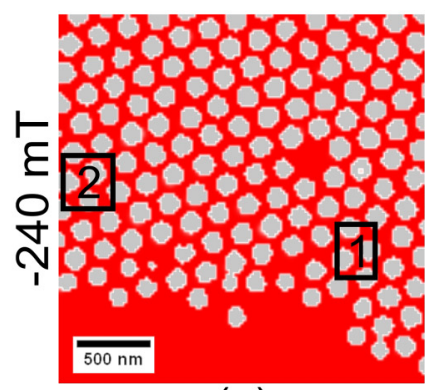

(e)

FIG. 4. SXM measurement series of a hexagonal antidot lattice (spacing $a=210 \mathrm{~nm}$ and diameter $d=160 \mathrm{~nm}$ ) in a $45 \mathrm{~nm}$ thin GdFe film. Images at an external applied field of (a) $240 \mathrm{mT}$ (positive saturation), (b) $0 \mathrm{mT}$, (c) $-8 \mathrm{mT}$ (close to the coercive field), (d) $-28 \mathrm{mT}$, and (e) $-240 \mathrm{mT}$ (negative saturation) are shown. A part of the antidot lattice in proximity of an unstructured area was purposely chosen for direct comparison with the native film in the same frame. Enlargements of the areas marked by black rectangles 1 and 2 are shown in Figs. 5 and 6 , respectively.

However, there is a broad distribution of interaction fields centered at $0 \mathrm{mT}$ spanning from $-14 \mathrm{mT}$ to $14 \mathrm{mT}$. Thus, there is no shift of the major hysteresis loop, as would be expected for an unbiased sample. The remanence, though, is reduced as the large negative interaction field overcomes the coercive field and leads to magnetization switching below zero field. Also, the saturation field is enlarged due to the large positive interaction field and the resulting increased external field required for some switching in comparison with the unstructured thin film. The absence of a wishbone structure in the FORC diagram further confirms that there is no significantly broad distribution of coercivities, hence, the coercivity of the native GdFe film is conserved and the change in magnetization reversal behavior is only due to a distribution of interaction fields [47]. This already indicates that the magnetization reversal for this particular antidot lattice geometry is not governed by long range movement of domain walls as these would be pinned at the constriction between two antidots. Pinning at the geometric structure, however, would lead to an increase of the coercive field compared to the intrinsic coercivity of the native material that is not observed [24,25].

To further elucidate the origin of the distribution of interaction fields SXM with XMCD contrast was employed to directly image the microscopic processes involved, shown in Fig. 4. To directly compare both the structured and unstructured magnetic GdFe film an image frame was chosen that shows both. At first glance the magnetization reversal appears to be chaotic and, indeed, individual switching events occur distributed over the whole antidot lattice at any given field. Turning to the x-ray micrograph at zero field, shown in Fig. 4(b), the effect of reduced remanence in the antidot lattice is clearly visible. While there is only a minimal part of the magnetization that has reversed in the unstructured part, a significant number of small reversed domains is found in the antidot lattice. These domains are uniformly distributed throughout the antidot lattice and have a size smaller than the antidot lattice unit cell. Hence, the whole antidot lattice area is exhibiting this reduction of remanence. As previously discussed, the coercivity is not affected by the nanostructuring and this is also confirmed in the $\mathrm{x}$-ray micrograph at the coercive field, shown in Fig. 4(c). Both antidot lattice and native thin film are approximately half reversed. However, it is noteworthy that the domain size in the antidot lattice is drastically decreased in comparison with the unstructured thin film. Going to higher fields, as shown in Fig. 4(d), the uniform thin film is already completely reversed. The antidot lattice, on the other hand, still features some small domains that have not yet switched, similar to the previously discussed situation at zero field, although with opposite sign of the stray field interaction. Again, these domains are distributed throughout the whole antidot lattice area and, thus, the increased saturation field is found throughout the structured area.

Looking at the antidot lattice more closely, two structural elements can be distinguished: a narrow material bridge between two adjacent holes and a node that connects its three neighboring bridges. From the SXM image at zero field, shown in Fig. 4(b), it becomes clear that there is no general preference for nodes or bridges to switch first, and they are equally found to be initially switched. Figure 5 shows an enlargement of position 1 of the previously discussed SXM measurement series, focusing on an initially switched bridge in the center. 

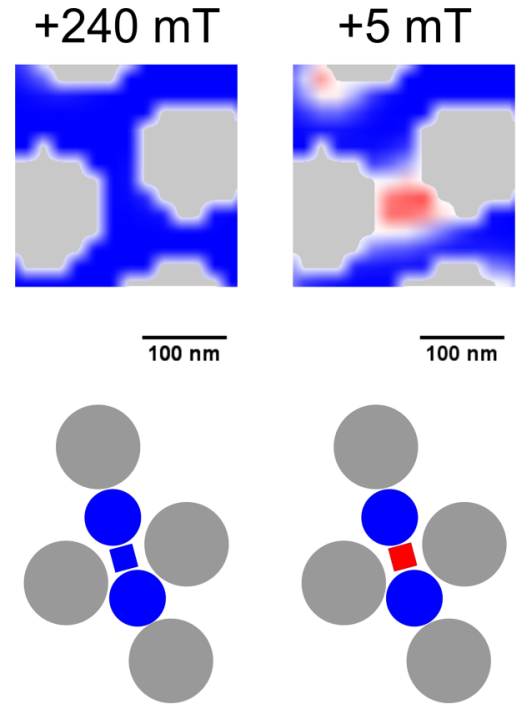
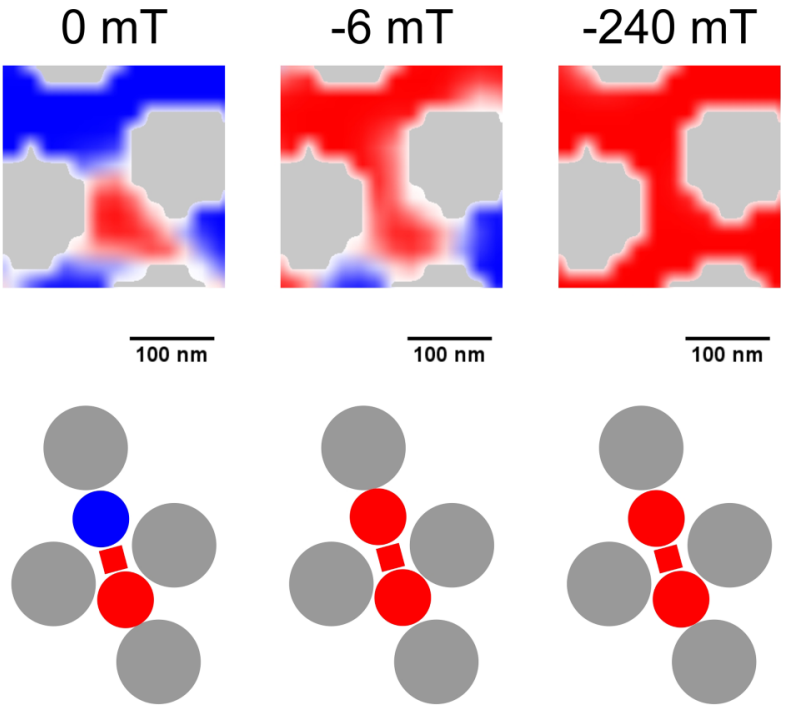

FIG. 5. Enlargement of position 1 of the SXM measurement series of a hexagonal antidot lattice (spacing $a=210 \mathrm{~nm}$ and diameter $d=160 \mathrm{~nm}$ ) in a $45 \mathrm{~nm}$ thin GdFe film shown in Fig. 4. Images at an external applied field of $240 \mathrm{mT}, 5 \mathrm{mT}, 0 \mathrm{mT},-6 \mathrm{mT}$, and -240 $\mathrm{mT}$ are shown. Additionally, schematic sketches of the magnetization states (up or down) of the central bridge and the two adjacent nodes are shown for each field.

During the magnetization reversal in this part of the antidot lattice this bridge switches first. It is noteworthy that the narrow bridge with a width of $50 \mathrm{~nm}$ switches separately from its two neighboring nodes and, thus, supports two domain walls. Subsequently, the lower node switches its magnetization state. Again, this small structure switches individually while the two other bridges connected to this node remain in their previous magnetization state. Finally, the upper node also switches and at increasing fields the magnetization is saturated.

Figure 6 focuses on position 2 of the previously discussed antidot lattice with an initially switched node in its center. Here, the central node switches first while the three neighboring bridges stay in their original magnetization state. It is noteworthy that this initial switching occurs at the same magnetic field as for the bridge centered in Fig. 5. In the area shown in Fig. 6 the magnetization configuration with only the central node switched is stable in a wide field range and only at an applied counter field of $-10 \mathrm{mT}$ the first neighboring bridge (lower left) switches. Subsequently, at $-14 \mathrm{mT}$ and $-24 \mathrm{mT}$, respectively, the other two bridges switch their magnetization state. The last bridge switches at a drastically increased magnetic field compared to the coercive field of the unstructured film, as it is stabilized in its original magnetization by the neighboring structure that has already reversed, thus, leading to an increase in saturation field of the antidot lattice.

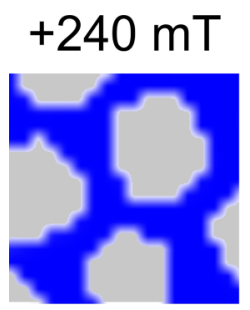

$\overline{100 \mathrm{~nm}}$

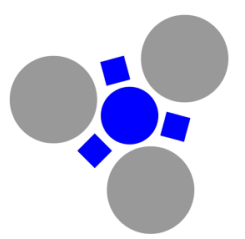

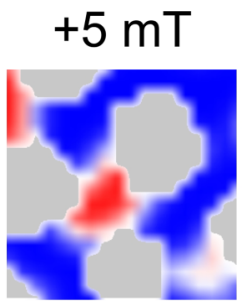

$\overline{100 \mathrm{~nm}}$

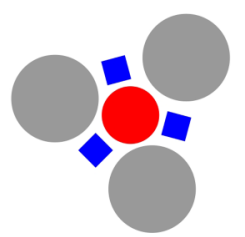

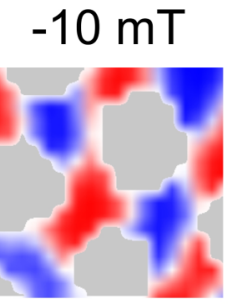

$\overline{100 \mathrm{~nm}}$

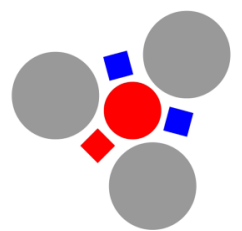

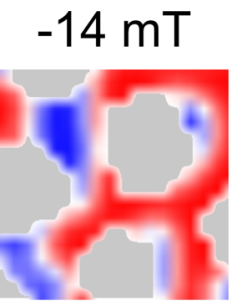

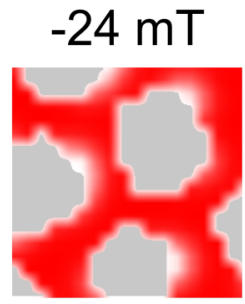

$\overline{100 \mathrm{~nm}}$
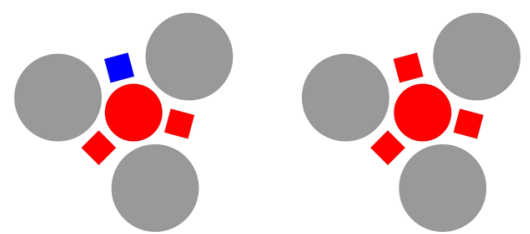
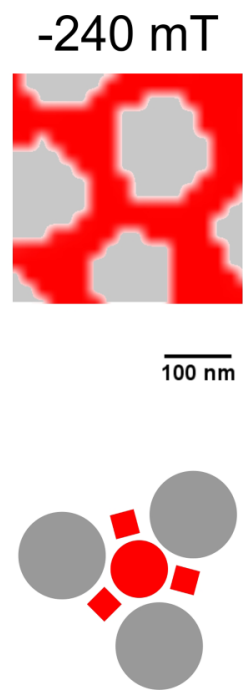

FIG. 6. Enlargement of position 2 of the SXM measurement series of a hexagonal antidot lattice (spacing $a=210 \mathrm{~nm}$ and diameter $d=160 \mathrm{~nm}$ ) in a $45 \mathrm{~nm}$ thin GdFe film shown in Fig. 4. Images at an external applied field of $240 \mathrm{mT}, 5 \mathrm{mT},-10 \mathrm{mT},-14 \mathrm{mT},-24 \mathrm{mT}$, and $-240 \mathrm{mT}$ are shown. Additionally, schematic sketches of the magnetization states (up or down) of the central node and the three adjacent bridges are shown for each field. 
Because the bridges are very narrow, the domain walls that are formed there can be short and, thus, the exchange energy associated with them is rather small. This is obvious from the fact that bridges and nodes can switch individually and thereby create multiple domain walls in a small area. Additionally, micromagnetic simulations confirm that one bridge can support two domain walls. However, a solely switched structural element is stabilized by the closure of stray fields with its neighboring elements. The same is true for the last element to switch; its original magnetization state is stabilized by stray field closure with its reversed neighbors. This stray field stabilization is also reproduced in micromagnetic simulations of a single pair of antidots that stabilize an individually switched bridge against strong external fields [46]. Hence, the stray field interaction is the microscopic origin of the interaction field $H_{u}$ that is detected in the FORC measurements. The distribution of interaction fields $H_{u}$ is due to the varying magnetization landscape throughout the antidot lattice. Each individual bridge has two neighboring nodes of which zero, one, or two can have the same magnetization state, and each node has three neighboring bridges of which zero, one, two, or three can have the same state. Thus, there is a large distribution of local interaction fields. From a stray field perspective the situation of the first and last element to switch is the same with opposing sign, hence, a symmetric distribution of $H_{u}$ centered at zero, as observed in the FORC measurement, is in agreement with this microscopic behavior.

Unfortunately, reproduction of the magnetization reversal process in micromagnetic simulation is difficult to achieve, because it requires careful balancing of the exchange stiffness and the saturation magnetization to allow the dipolar interaction to overcome the exchange interaction and to enforce two domain walls in the constriction between to antidots. Moreover, defects in the magnetization were introduced in the simulations to cope with the numerically difficult and time consuming reversal processes. Furthermore, simulation of the statistically distributed switchings of nodes and bridges requires the investigation of a large antidot lattice with small field steps, which is computationally prohibitive. While important features like the formation of two domain walls in one bridge and the stabilization of these small domains against external fields were confirmed in micromagnetic simulations, perfect reproduction of the full magnetization reversal process was not achieved.

It can be concluded that for this antidot lattice geometry the magnetization reversal mechanism is dominated by stray field interaction between neighboring structure elements that switch individually. The stray field interaction can overcome the exchange energy needed for domain wall formation, because the bridges are very narrow and allow equally short domain walls.

\section{B. Antidot lattices with small holes}

The FORC diagram for a hexagonal antidot lattice with small hole diameter (antidot spacing $a=210 \mathrm{~nm}$ and diameter $d=80 \mathrm{~nm}$ ) is shown in Fig. 7 with the major hysteresis loop shown as inset. There is a fundamental difference between the present FORC diagram and that obtained for large antidot

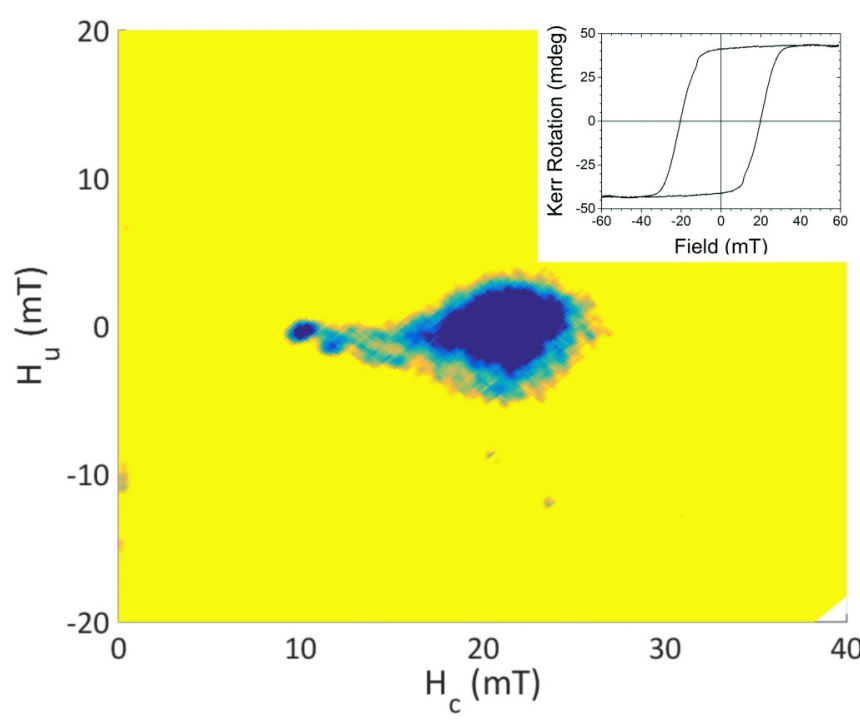

FIG. 7. FORC density of a hexagonal antidot lattice (antidot spacing $a=210 \mathrm{~nm}$ and diameter $d=80 \mathrm{~nm}$ ) in a $29 \mathrm{~nm}$ thin GdFe film. The major hysteresis loop is shown as inset.

diameters (cf. Sec. III A and Fig. 3), already hinting at a different microscopic magnetization reversal mechanism. In contrast to the geometry with large holes, there is only a narrow distribution of interaction fields $H_{u}$. However, there is a broad distribution of coercive fields $H_{c}$ starting at $10 \mathrm{mT}$ and leading up to $25 \mathrm{mT}$. While the lowest field value of the $H_{c}$ distribution coincides with the coercive field of the native GdFe film, the $H_{c}$ distribution indicates irreversible processes extending up to fields more than doubled as compared to unpatterned samples. This indicates that for this antidot lattice geometry the nanostructuring results in a significant increase of the overall coercive field. Unlike for large holes, here, there is no significant negative interaction field that results in irreversible processes occurring at lower fields than $H_{c}$, thus, the slight reduction in remanence compared to the uniform film can be attributed to reversible magnetization rotation.

To reveal the origin of this coercive enhancement SXM imaging with XMCD contrast has been employed and a selection of magnetic field steps are shown in Fig. 8. Like the FORC diagram, the SXM measurements indicate a completely different magnetization reversal behavior for the antidot lattice geometry with small holes in comparison with the previously discussed geometry with large holes. First, domains appear to nucleate [Fig. 8(b)] at locations which, due to antidot lattice defects, locally resemble an unpatterned film. In contrast to the previously discussed geometry all magnetic domains are larger than the antidot lattice unit cell, thus, bridges and nodes do not switch individually. However, the domain walls are located at the constrictions between two holes to reduce the domain wall length and the associated exchange energy in this geometry as well. Subsequently, the domain walls propagate [Figs. 8(c)$8(\mathrm{e})]$ in an increasing magnetic field. During propagation the domain walls get pinned, depinned, and repinned at different positions in the antidot lattice. The pinning strength between different holes depends on the length of the domain wall at this position that is determined by the spacing between the two adjacent holes [20]. As nanosphere lithography results in 


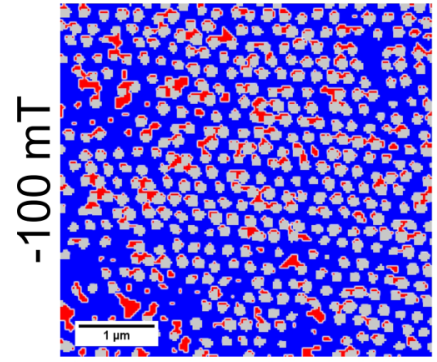

(a)

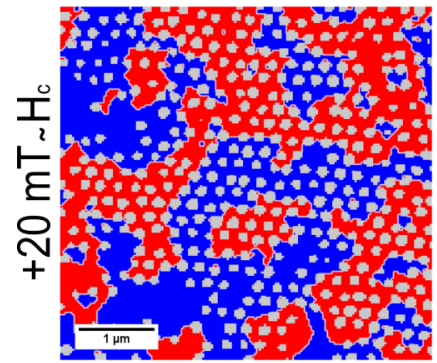

(d)

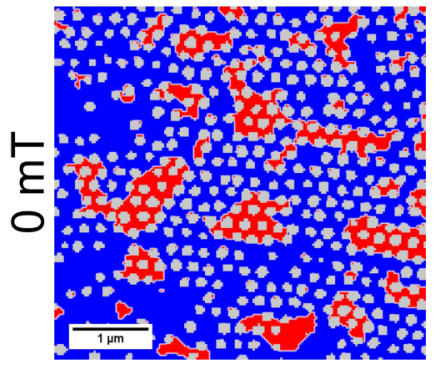

(b)

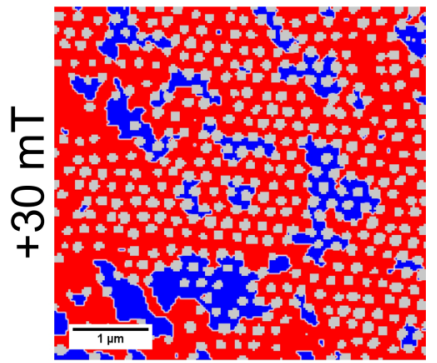

(e)

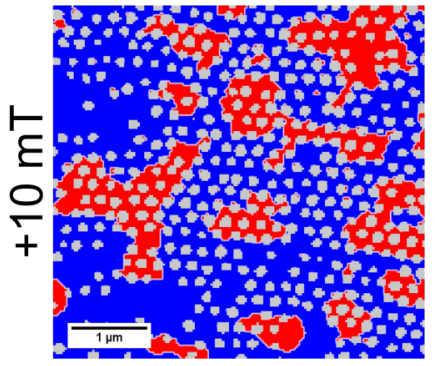

(c)

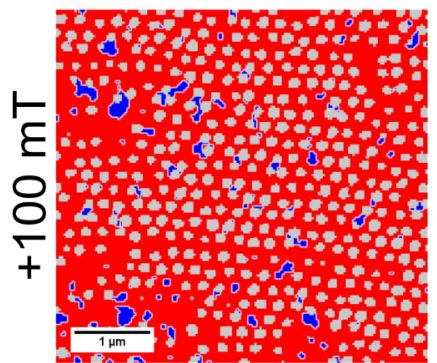

(f)

FIG. 8. SXM measurement series of a hexagonal antidot lattice (spacing $a=210 \mathrm{~nm}$ and diameter $d=80 \mathrm{~nm}$ ) in a $29 \mathrm{~nm}$ thin GdFe film. Images at an external applied field of (a) - $100 \mathrm{mT}$ (negative saturation), (b) $0 \mathrm{mT}$, (c) $10 \mathrm{mT}$, (d) $20 \mathrm{mT}$ (close to the coercive field), (e) $30 \mathrm{mT}$, and (f) $100 \mathrm{mT}$ (positive saturation) are shown.

a somewhat broad distribution of hole diameters and spacings [37], there is a corresponding distribution of the pinning strength at these holes. In turn, this leads to the observed distribution in coercive field $H_{c}$.
This magnetization reversal mechanism for antidot lattices with small hole diameter is further confirmed by micromagnetic simulation of a lattice with spacing $a=220 \mathrm{~nm}$ and diameter $d=95 \mathrm{~nm}$ shown in Fig. 9. Qualitatively, the

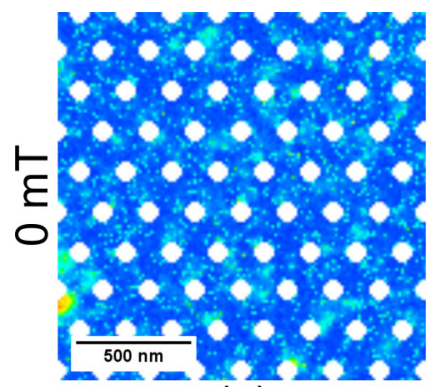

(a)

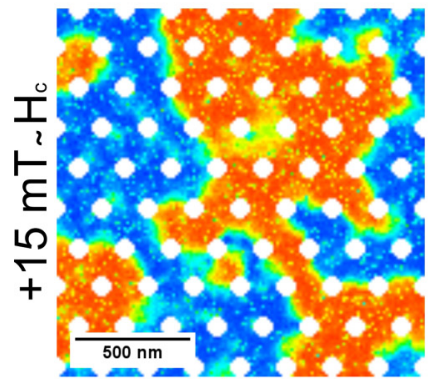

(d)

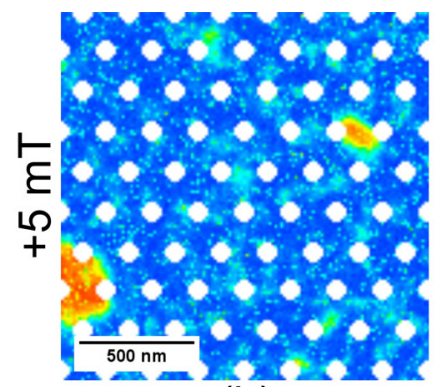

(b)

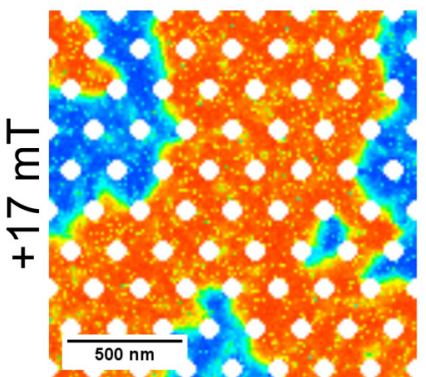

(e)

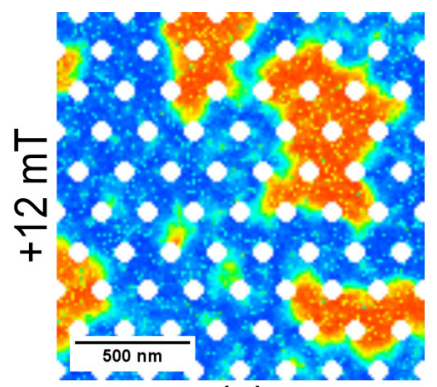

(c)

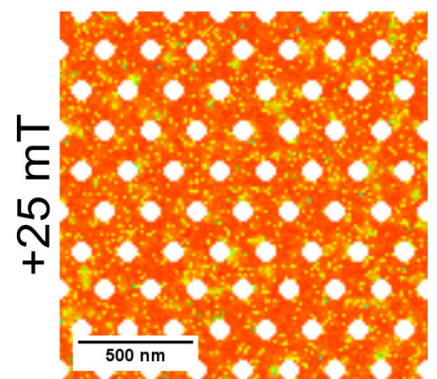

(f)

FIG. 9. Simulation of the magnetization reversal of a hexagonal antidot lattice (spacing $a=220 \mathrm{~nm}$ and diameter $d=95 \mathrm{~nm}$ ) in a $28 \mathrm{~nm}$ thin GdFe film. Images at an external applied field of (a) $0 \mathrm{mT}$, (b) $5 \mathrm{mT}$, (c) $12 \mathrm{mT}$, (d) $15 \mathrm{mT}$ (close to the coercive field), (e) $17 \mathrm{mT}$, and (f) $25 \mathrm{mT}$ (positive saturation) are shown. 
simulation is in excellent agreement with the experimental images shown in Fig. 8. At low fields domains nucleate [Fig. 9(b)] and, subsequently, the domain walls propagate [Figs. 9(c)-9(e)] through the antidot lattice. During the propagation stage the domain walls get pinned, depinned, and repinned, and the magnetization reversal occurs by the same mechanism as in the experiment. However, the field range for the domain wall propagation stage is much smaller than in the experiment, because the antidot lattice order is lower and the distribution of antidot spacings is much broader in real samples. Indeed, experimentally a much narrower distribution of coercive fields is observed for antidot lattices with improved order (not shown here). Additionally, the micromagnetic simulations also reproduce the fact that domain walls are always spanning the constriction between two holes to decrease domain wall length.

For the antidot lattice geometry with small holes and, thus, wider material bridges it can be summarized that the magnetization reversal follows a domain wall nucleation and propagation mechanism with the domain walls always being located between two neighboring holes. This is similar to the magnetization reversal mechanism observed for antidot lattices in in-plane magnetized materials [15,17,19,21,24]. Here, the exchange interaction is the dominating influence, enforcing a smaller number of larger domains.

\section{CONCLUSION}

For soft-magnetic GdFe thin films with perpendicular magnetic anisotropy hosting hexagonal antidot lattices we find two regimes of magnetization reversal processes depending on the geometric properties of the nanostructures. As it turns out, the most relevant parameter is the material width between two neighboring holes as this establishes the length of the domain walls in the antidot lattices. The magnetization reversal processes are either dominated by the exchange interaction or the dipolar interaction, both of which can be tailored by tuning the geometry of the antidot arrangement. In this way, different aspects of the magnetic properties can be altered. Even after nanopatterning both regimes are still influenced by the coercive field of the native film. In the dipole dominated regime the coercive field is preserved, and in the exchange dominated regime it determines the lower limit of the switching field distribution.

For comparably wide bridges between the antidots and, thus, longer domain walls, a magnetization reversal similar to in-plane magnetized materials hosting antidot lattices is found. Magnetization reversal is achieved by domain nucleation and subsequent domain wall movement and pinning, depinning, and repinning at a different site. The constrictions between the antidots act as pinning centers and the distribution of bridge widths is directly reflected in the distribution of coercive fields. The driving mechanism in this case is the reduction of the domain wall length when it is located between holes compared to the unpatterned material. Hence, this geometric regime can be used to increase the coercive field of the system in comparison with the as-prepared films.

However, the enhancement of the coercive field is limited when further decreasing the bridge width. At some point the domain walls get short enough for the gain in dipole energy by stray field closure to be larger than the exchange energy loss associated with the domain walls. In the regime of very small bridge widths a single constriction between two antidots can hold two domain walls. The bridges and nodes can then switch independently during magnetization reversal and the magnetic domains may become smaller than the antidot lattice unit cell. Because there is abrupt individual switching of local elements, there is no domain wall movement during magnetization reversal that could be hindered by the antidots as pinning centers. Thus, the coercive field returns to the value of the unpatterned material in the dipole dominated regime. Due to individual switching of bridges and nodes there is a broad variety of local stray field landscapes that can either lead to stabilization or destabilization of the magnetization state of an individual element. This results in the reduction of the remanent magnetization, the increase of the saturation field, and a broad switching field distribution due to a broad interaction field distribution. Although there is no coercivity enhancement, the stray field closure can stabilize very small domains in a wide range of external magnetic fields. This could be the basis for the realization of a bit patterned media.

The FORC measurements allowed us to distinguish the origin of the switching field distributions and indicate different microscopic magnetization reversal processes without the need for high lateral resolution that would not have been possible from the major hysteresis loops alone. For the exchange dominated regime a coercive field distribution, and for the dipole dominated regime an interaction field distribution is found and confirmed by SXM imaging. Hence, nanostructures in a softmagnetic thin film with perpendicular magnetic anisotropy are a model case where FORC measurements reliably quantify the microscopic interactions and the FORC distributions are directly interpretable. However, the microscopic origin of these interactions and the actual reversal mechanism could not be elucidated without spatially resolving measurements.

\section{ACKNOWLEDGMENTS}

The authors would like to thank Michael Bechtel for support during beam times and Bernd Ludescher for thin film deposition. Furthermore, we are grateful to Ulrike Eigenthaler for performing SEM measurements. Helmholtz Zentrum Berlin is acknowledged for allocating beam time at the BESSY II synchrotron radiation facility. Financial support by the Baden-Württemberg Stiftung in the framework of the Kompetenznetz Funktionelle Nanostrukturen is gratefully acknowledged.
[1] M. J. Pechan, C. Yu, R. L. Compton, J. P. Park, and P. A. Crowell, J. Appl. Phys. 97, 10J903 (2005).

[2] B. Lenk, H. Ulrichs, F. Garbs, and M. Münzenberg, Phys. Rep. 507, 107 (2011).
[3] H. Yu, G. Duerr, R. Huber, M. Bahr, T. Schwarze, F. Brandl, and D. Grundler, Nat. Commun. 4, 2702 (2013).

[4] R. P. Cowburn, A. O. Adeyeye, and J. A. C. Bland, Appl. Phys. Lett. 70, 2309 (1997). 
[5] L. Torres, L. Lopez-Diaz, and J. Iñiguez, Appl. Phys. Lett. 73, 3766 (1998).

[6] M. B. A. Jalil, J. Appl. Phys. 93, 7053 (2003).

[7] J. P. Morgan, A. Stein, S. Langridge, and C. H. Marrows, Nat. Phys. 7, 75 (2010).

[8] F. Haering, U. Wiedwald, T. Häberle, L. Han, A. Plettl, B. Koslowski, and P. Ziemann, Nanotechnology 24, 055305 (2013).

[9] L. J. Heyderman, Nat. Nanotechnol. 8, 705 (2013).

[10] M. F. Laguna, C. A. Balseiro, D. Domínguez, and F. Nori, Phys. Rev. B 64, 104505 (2001).

[11] E. Mengotti, L. J. Heyderman, A. F. Rodriguez, F. Nolting, R. V. Hügli, and H. B. Braun, Nat. Phys. 7, 68 (2011).

[12] J. Gräfe, F. Haering, T. Tietze, P. Audehm, M. Weigand, U. Wiedwald, P. Ziemann, P. Gawroński, G. Schütz, and E. J. Goering, Nanotechnology 26, 225203 (2015).

[13] F. J. Castano, K. Nielsch, C. A. Ross, J. W. A. Robinson, and R. Krishnan, Appl. Phys. Lett. 85, 2872 (2004).

[14] C. C. Wang, A. O. Adeyeye, and N. Singh, Nanotechnology 17, 1629 (2006).

[15] F. Haering, U. Wiedwald, S. Nothelfer, B. Koslowski, P. Ziemann, L. Lechner, A. Wallucks, K. Lebecki, U. Nowak, J. Gräfe, E. Goering, and G. Schütz, Nanotechnology 24, 465709 (2013).

[16] C. C. Wang, A. O. Adeyeye, and Y. H. Wu, J. Appl. Phys. 94, 6644 (2003).

[17] L. J. Heyderman, F. Nolting, D. Backes, S. Czekaj, L. LopezDiaz, M. Kläui, U. Rüdiger, C. A. F. Vaz, J. A. C. Bland, R. J. Matelon, U. G. Volkmann, and P. Fischer, Phys. Rev. B 73, 214429 (2006).

[18] G. Ctistis, E. Papaioannou, P. Patoka, J. Gutek, P. Fumagalli, and M. Giersig, Nano Lett. 9, 1 (2009).

[19] F. Béron, K. R. Pirota, V. Vega, V. M. Prida, A. Fernández, B. Hernando, and M. Knobel, New J. Phys. 13, 013035 (2011).

[20] P. Gawroński, K. J. Merazzo, O. Chubykalo-Fesenko, A. Asenjo, R. P. del Real, and M. Vázquez, Europhys. Lett. 100, 17007 (2012).

[21] K. J. Merazzo, C. Castan-Guerrero, J. Herrero-Albillos, F. Kronast, F. Bartolome, J. Bartolome, J. Sese, R. P. del Real, L. M. Garcia, and M. Vazquez, Phys. Rev. B 85, 184427 (2012).

[22] M. P. Proenca, K. J. Merazzo, L. G. Vivas, D. C. Leitao, C. T. Sousa, J. Ventura, J. P. Araujo, and M. Vazquez, Nanotechnology 24, 475703 (2013).

[23] S. Mallick and S. Bedanta, J. Magn. Magn. Mater. 382, 158 (2015).

[24] J. Gräfe, M. Weigand, C. Stahl, N. Träger, M. Kopp, G. Schütz, E. J. Goering, F. Haering, P. Ziemann, and U. Wiedwald, Phys. Rev. B 93, 014406 (2016).

[25] D. Tripathy and A. O. Adeyeye, New J. Phys. 13, 023035 (2011).

[26] Y.-C. Huang, J.-C. Hsiao, I.-Y. Liu, L.-W. Wang, J.-W. Liao, and C.-H. Lai, J. Appl. Phys. 111, 07 B923 (2012).
[27] F. Béron, L.-P. Carignan, D. Ménard, and A. Yelon, Electrodeposited Nanowires and their Applications (Intech, 2010) Chap. Extracting Individual Properties from Global Behaviour: Firstorder Reversal Curve Method Applied to Magnetic Nanowire Arrays.

[28] C. R. Pike, A. P. Roberts, and K. L. Verosub, J. Appl. Phys. 85, 6660 (1999).

[29] A. P. Roberts, C. R. Pike, and K. L. Verosub, J. Geophys. Res., [Solid Earth Planets] 105, 28461 (2000).

[30] R. J. Harrison and J. M. Feinberg, Geochem. Geophys. Geosys. 9, Q05016 (2008).

[31] J. E. Davies, O. Hellwig, E. E. Fullerton, J. S. Jiang, S. D. Bader, G. T. Zimányi, and K. Liu, Appl. Phys. Lett. 86, 262503 (2005).

[32] C.-I. Dobrotă and A. Stancu, J. Appl. Phys. 113, 043928 (2013).

[33] C.-I. Dobrotă and A. Stancu, Physica B: Condensed Matter 457, 280 (2015).

[34] J. E. Davies, O. Hellwig, E. E. Fullerton, G. Denbeaux, J. B. Kortright, and K. Liu, Phys. Rev. B 70, 224434 (2004).

[35] D. Navas, J. Torrejon, F. Béron, C. Redondo, F. Batallan, B. P. Toperverg, A. Devishvili, B. Sierra, F. Castaño, K. R. Pirota, and C. A. Ross, New J. Phys. 14, 113001 (2012).

[36] A. Plettl, F. Enderle, M. Saitner, A. Manzke, C. Pfahler, S. Wiedemann, and P. Ziemann, Adv. Funct. Mater. 19, 3279 (2009).

[37] U. Wiedwald, F. Haering, S. Nau, C. Schulze, H. Schletter, D. Makarov, A. Plettl, K. Kuepper, M. Albrecht, J. Boneberg, and P. Ziemann, Beilstein J. Nanotechnol. 3, 831 (2012).

[38] E. Amaladass, B. Ludescher, G. Schütz, T. Tyliszczak, and T. Eimüller, Appl. Phys. Lett. 91, 172514 (2007).

[39] R. Davies, D. Edwards, J. Gräfe, L. Gilbert, P. Davies, G. Hutchings, and M. Bowker, Surf. Sci. 605, 1754 (2011).

[40] J. Gräfe, M. Schmidt, P. Audehm, G. Schütz, and E. Goering, Rev. Sci. Instrum. 85, 023901 (2014).

[41] D. Nolle, M. Weigand, P. Audehm, E. Goering, U. Wiesemann, C. Wolter, E. Nolle, and G. Schütz, Rev. Sci. Instrum. 83, 046112 (2012).

[42] W. Rasband, ImageJ, U.S. National Institutes of Health, Bethesda, Maryland, USA, http://imagej.nih.gov/ij/, 19972015.

[43] P. Thevenaz, U. E. Ruttimann, and M. Unser, IEEE Trans. Image Process. 7, 27 (1998).

[44] N. Kazantseva, D. Hinzke, U. Nowak, R. W. Chantrell, U. Atxitia, and O. Chubykalo-Fesenko, Phys. Rev. B 77, 184428 (2008).

[45] R. F. L. Evans, D. Hinzke, U. Atxitia, U. Nowak, R. W. Chantrell, and O. Chubykalo-Fesenko, Phys. Rev. B 85, 014433 (2012).

[46] M. Skripnik, Controlling the Magnetic Structure in Antidot Arrays: A Numerical Study, Master's thesis, University of Konstanz, 2014.

[47] C. R. Pike, C. A. Ross, R. T. Scalettar, and G. Zimanyi, Phys. Rev. B 71, 134407 (2005). 